Cahiers
de ea Recherche
sur les Droits Fondamentaux
Cahiers de la recherche sur les droits fondamentaux

$8 \mid 2010$

La liberté d'expression

\title{
Chronique de jurisprudence constitutionnelle française 2009
}

Xavier Aurey, Aurore Catherine, Anne-Sophie Denolle, Marie Rota, Vincent Souty et Antoine Siffert

\section{(2) OpenEdition}

1 Journals

Édition électronique

URL : https://journals.openedition.org/crdf/6192

DOI : $10.4000 /$ crdf.6192

ISSN : 2264-1246

Éditeur

Presses universitaires de Caen

Édition imprimée

Date de publication : 20 décembre 2010

Pagination : 149-162

ISBN : 978-2-84133-367-7

ISSN : 1634-8842

Référence électronique

Xavier Aurey, Aurore Catherine, Anne-Sophie Denolle, Marie Rota, Vincent Souty et Antoine Siffert, "Chronique de jurisprudence constitutionnelle française 2009 ", Cahiers de la recherche sur les droits fondamentaux [En ligne], 8 | 2010, mis en ligne le 08 octobre 2020, consulté le 14 novembre 2022. URL : http://journals.openedition.org/crdf/6192 ; DOI : https://doi.org/10.4000/crdf.6192 


\title{
Chronique de jurisprudence constitutionnelle française $2009^{1}$
}

\author{
I. Les techniques de contrôle \\ A. Intelligibilité de la loi et sincérité et clarté du débat parlementaire \\ B. La séparation des pouvoirs \\ C. Les réserves d'interprétation du Conseil constitutionnel
}

II. Le contrôle substantiel

A. Le principe d'égalité

1. Le principe d'égalité et la "contribution carbone "

2. Le principe d'égalité et la question prioritaire de constitutionnalité

B. La protection du droit d'auteur et l'accès à Internet

De mars 2009 à décembre de cette même année, le Conseil constitutionnel a consacré une partie substantielle de sa jurisprudence à l'examen des textes permettant l'application de la réforme constitutionnelle du 23 juillet 2008. À ce titre, le Conseil dégage d'ailleurs un nouvel objectif de valeur constitutionnelle ${ }^{2}$, celui de l'indépendance des médias ${ }^{3}$, directement issu de la réforme de 2008 et de la nouvelle rédaction de l'article 34 de la Constitution qui renvoie à « la liberté, le pluralisme et l'indépendance des médias ${ }^{4}$. Par ailleurs, dans la décision concernant l'importante loi organique relative à la question prioritaire de constitutionnalité, issue du nouvel article 61-1 de la Constitution, le Conseil qualifie pour la première fois la bonne administration de la justice d'objectif de valeur constitutionnelle (OVC) ${ }^{5}$.

Autre nouveauté, dans sa décision $\mathrm{n}^{\circ} 2009-579^{6}$, le Conseil semble opérer un revirement en ce qu'il vise, au même titre que les observations du Gouvernement, les observations présentées par soixante-dix-neuf députés, alors qu'il les déclarait irrecevables depuis une décision

1. Chronique réalisée en collaboration par Xavier Aurey, Aurore Catherine, Anne-Sophie Denolle (attachés temporaires d'enseignement et de recherche à l'Université de Caen Basse-Normandie), Marie Rota, Vincent Souty (attachés temporaires d'enseignement et de recherche à l'Université du Havre) et Antoine Siffert (doctorant à l'Université du Havre).

2. Sur cette question des OVC, voir notre première "Chronique de jurisprudence constitutionnelle française janvier 2007-mars 2008 ", Les cahiers de la recherche sur les droits fondamentaux, $\mathrm{n}^{\circ}$ 6, 2008, p. 175 sq.

3. C.C., déc. $\mathrm{n}^{0}$ 2009-577 DC du 3 mars 2009, Loi relative à la communication audiovisuelle et au nouveau service public de la télévision (ci-après déc. Communication audiovisuelle), cons. 3 .

4. Sur ce point: R. Ghevontian, S. Lamouroux, O. Le Bot et E. Kerviche, «Jurisprudence du Conseil constitutionnel», RFDC, n 79, 2009/3, p. 590; L. Baghestani-Perrey, A.-L. Cassard-Valembois et L. Janicot, "Chronique de jurisprudence constitutionnelle $\mathrm{n}^{\circ} 7$ ", Petites Affiches, $\mathrm{n}^{\circ}$ 153, $2009, \mathrm{p} .11$.

5. C.C., déc. $\mathrm{n}^{\circ}$ 2009-595 DC du 3 décembre 2009, Loi organique relative à l'application de l'article 61-1 de la Constitution, cons. 4 (ci-après déc. Loi organique 61-1).

6. C.C., déc. $\mathrm{n}^{\circ}$ 2009-579 DC du 9 avril 2009, Loi organique relative à l'application des articles 34-1, 39 et 44 de la Constitution. 
de $1992^{7}$, un revirement qui s'explique lui aussi, selon Lætitia Janicot, par la nouvelle procédure d'examen $a$ posteriori de la conformité des lois à la Constitution ${ }^{8}$.

L'influence de la réforme de 2008 s'est également fait sentir dans les techniques de contrôle du Conseil (I) qui en a profité pour affiner sa jurisprudence relative aux principes d'intelligibilité de la loi et de sincérité et clarté du débat parlementaire $(\mathrm{A})$, au principe de séparation des pouvoirs $(\mathrm{B})$, tout en faisant une utilisation très large de ses réserves d'interprétation $(\mathrm{C})$. Néanmoins, on ne peut réduire l'intérêt de l'étude de la jurisprudence du Conseil aux seuls aspects formels. Des décisions importantes concernant la substance des droits protégés par la Constitution ont été adoptées (II), qui concernent tant le principe d'égalité (A) que la lutte contre la contrefaçon sur Internet (B).

\section{Les techniques de contrôle}

\section{A. Intelligibilité de la loi et sincérité et clarté du débat parlementaire}

Le principe de clarté et de sincérité du débat parlementaire et l'objectif d'intelligibilité et d'accessibilité de la loi s'inscrivent tous deux dans l'optique de la consolidation de l'État de droit démocratique. Le premier doit permettre l'émergence de la volonté générale à travers l'expression d'un débat où les partis d'opposition pourront faire entendre leur voix ; le second concerne la possibilité pour le citoyen de comprendre la norme et de participer aussi au respect de l'État de droit. Le Conseil constitutionnel s'adresse alors au législateur et s'intéresse au travail parlementaire d'élaboration de la loi.

Consacré d'abord dans la décision $\mathrm{n}^{\circ}$ 99-421 du 16 décembre 1999, l'objectif d'intelligibilité et d'accessibilité de la loi est très fréquemment invoqué par les requérants devant le Conseil et relié au problème de l'incompétence négative, les parlementaires requérants estimant que lorsque le pouvoir législatif renvoie le soin au pouvoir réglementaire de compléter la norme, cela nuit à l'intelligibilité de la loi. Le Conseil le rappelle d'ailleurs dans la décision $\mathrm{n}^{\circ}$ 2009-592 portant sur la loi relative à l'orientation et à la formation professionnelles tout au long de la vie, il s'agit de "prémunir les sujets de droit contre une interprétation contraire à la Constitution ou contre le risque d'arbitraire, sans reporter sur des autorités administratives ou juridictionnelles le soin de fixer des règles dont la détermination n'a été confiée par la Constitution qu’à la loi » ${ }^{9}$.

C'est ainsi que le Conseil censure, dans la décision $\mathrm{n}^{\circ}$ 2009-584, loi portant réforme de l'hôpital et relative aux patients, à la santé et aux territoires, l'article 17 de la loi, qui renvoyait à la Cour des comptes la coordination des modalités de certifications des établissements de santé par les commissaires aux comptes, coordination elle-même fixée par voie réglementaire ${ }^{10}$. Le Conseil se fonde sur l'OVC d'intelligibilité et d'accessibilité de la loi pour censurer cette disposition qui ne permet pas de fixer les limites du pouvoir de la Cour des comptes. Il en profite pour rappeler le fondement textuel auquel il se rattache pour justifier cet OVC: il s'agit des articles 4, 5, 6 et 16 de la Déclaration des Droits de l'homme et du citoyen de 1789 (DDHC).

Dans les autres décisions étudiées, le Conseil se contente de rappeler la signification concrète de l'OVC et rappelle que l'intelligibilité et l'accessibilité de la loi imposent au législateur d'adopter « des dispositions suffisamment précises et des formules non équivoques ${ }^{11}$; il rejette le grief des requérants en considérant que les dispositions contestées ne portent pas atteinte à cet objectif: dans la décision $\mathrm{n}^{0}$ 2009-580, la distinction entre l'obligation de surveillance de l'accès à Internet et le délit de contrefaçon ${ }^{12}$; dans la décision $\mathrm{n}^{\mathrm{0}} 2009-588$, celle entre commune touristique et zone touristique ${ }^{13}$; dans la décision $\mathrm{n}^{\circ}$ 2009-594, l'imprécision de la loi ne méconnaît pas l'objectif d'intelligibilité de la loi dès lors que cette même loi fait référence à une convention pluriannuelle de rémunération signée entre la RATP et le Syndicat des transports d'Île-de-France qui permettra de clarifier et déterminer les obligations réciproques des cocontractants ${ }^{14}$.

Le principe de clarté et de sincérité du débat parlementaire est récent, puisque l'expression apparaît dans la décision $n^{0} 2005-526$ du 13 octobre 2005, même si on peut relever une première occurrence, annonciatrice du principe, dans la décision $\mathrm{n}^{\mathrm{0}} 2005-512$ du mois d'avril $2005^{15}$. On en observe néanmoins une utilisation croissante, notamment au cours de la période étudiée. Le Conseil est

7. C.C., déc. nº 92-305 DC du 21 février 1992.

8. L. Baghestani-Perrey, A.-L. Cassard-Valembois et L. Janicot, «Chronique de jurisprudence constitutionnelle $\mathrm{n}^{\circ} 7$ (suite et fin) », Petites Affiches, $\mathrm{n}^{\circ} 155$ 2009, p. 9 sq.: «La révision constitutionnelle du 23 juillet 2008 ne rendait pas nécessaire cette évolution, puisqu'elle n’impose le respect des exigences du procès équitable que dans le cadre du contrôle a posteriori des lois. Mais, elle l'explique peut-être, dans la mesure où elle devrait conduire à renouveler les réflexions qui ont déjà pu être engagées sur la procédure suivie devant le Conseil, auxquelles ce dernier ne peut que prendre part ».

9. C.C., déc. $\mathrm{n}^{\mathrm{o}}$ 2009-592 DC du 19 novembre 2009, Loi relative à l'orientation et à la formation professionnelle tout au long de la vie, cons. 6.

10. C.C., déc. $\mathrm{n}^{\circ}$ 2009-584 DC du 16 juillet 2009, Loi portant réforme de l'hôpital et relative aux patients, à la santé et aux territoires (ci-après déc. Loi HPST), cons. 29.

11. C.C., déc. $\mathrm{n}^{\circ}$ 2009-578 DC du 18 mars 2009, Loi de mobilisation pour le logement et de lutte contre l'exclusion, cons. 24

12. C.C., déc. $\mathrm{n}^{\circ}$ 2009-580 DC du 10 juin 2009, Loi favorisant la diffusion et la protection de la création sur Internet (ci-après déc. Hadopi I), cons. 7.

13. C.C., déc. $\mathrm{n}^{\circ}$ 2009-588 DC du 6 août 2009, Loi réaffirmant le principe du repos dominical et visant à adapter les dérogations à ce principe dans les communes et zones touristiques et thermales ainsi que dans certaines grandes agglomérations pour les salariés volontaires (ci-après déc. Repos dominical), cons. 7 .

14. C.C., déc. $\mathrm{n}^{\circ}$ 2009-594 DC du 3 décembre 2009, Loi relative à la régulation des transports ferroviaires et portant diverses dispositions relatives aux transports (ci-après déc. Transports ferroviaires), cons. 11.

15. C.C., déc. $\mathrm{n}^{\circ}$ 2005-526 DC du 13 octobre 2005, Résolution modifiant le règlement de l’Assemblée nationale, cons. 5 et C.C., déc. $\mathrm{n}^{\circ} 2005-512$ DC du 21 avril 2005, Loi d'orientation et de programme pour l'avenir de l'école, cons. 4. 
en effet venu préciser sa jurisprudence et a censuré à plusieurs reprises les textes qui lui étaient soumis au nom du respect du principe de clarté et de sincérité du débat parlementaire.

Il apparaît même en tant que norme de référence ${ }^{16}$ des décisions $n^{\circ} 2009-581$ et $n^{\circ} 2009-582$ qui portent sur des résolutions tendant à modifier les règlements respectivement de l'Assemblée nationale et du Sénat. Le Conseil vient ici poser à deux reprises un considérant de principe, repris à l'identique dans les deux décisions, dans lequel il confirme le rattachement du principe de clarté et de sincérité du débat parlementaire à une lecture combinée des articles 6 de la DDHC et 3 de la Constitution, un rattachement, assez artificiel, illustrant, sans doute, un réflexe positiviste dont on pourrait finalement discuter l'opportunité ${ }^{17}$.

L'affirmation de ce principe a pour but principal de permettre à l'opposition de s'exprimer lors des débats, on se rattache ici à l'idée du pluralisme de l'expression des idées politiques, l'instauration d'un véritable débat étant propice à l'émergence de la volonté générale.

Dans la décision $n^{\circ}$ 2009-581 portant sur la résolution tendant à modifier le règlement de l'Assemblée, le Conseil prononce ainsi la censure de l'article 38 de cette résolution en soulevant le fait qu'elle aurait pu avoir pour conséquence d'interdire aux groupes d'opposition de s'exprimer ${ }^{18}$. Par ailleurs, si le Conseil valide les différentes dispositions visant à limiter le temps de parole des orateurs à deux minutes, c'est sous la réserve que le président de séance applique cette limite en «veillant au respect des exigences de clarté et de sincérité du débat parlementaire» ${ }^{19}$.

D'autres réserves d'interprétation sont également posées en vue du respect du principe de clarté et de sincérité du débat parlementaire. Ainsi, l'article 26 de la résolution qui modifie les conditions dans lesquelles les commissions parlementaires organisent la publicité de leurs travaux et modifie l'article $46 \mathrm{du}$ règlement de l'Assemblée doit être lu à la lumière de ce principe de clarté et de sincérité et le Conseil précise que le travail en commission doit faire l'objet d'un compte rendu détaillé $^{20}$. En outre, toujours en ce qui concerne le travail en commission, le Conseil précise que la possibilité reconnue au président de la commission de déroger au délai de droit commun pour le dépôt des amendements devra être conciliée avec les exigences du principe de clarté et de sincérité du débat parlementaire ${ }^{21}$.

Dans la décision $\mathrm{n}^{0}$ 2009-582, résolution tendant à la modification du règlement du Sénat, le Conseil ne censure aucune disposition sur la base du non-respect de ce principe. Néanmoins, il n'est pas totalement absent puisque l'article 15 de la résolution est déclaré contraire à la Constitution en tant qu'il empêcherait la tenue d'un débat d'orientation en séance publique sur un projet de texte avant son examen par la commission à laquelle il a été renvoyé ${ }^{22}$.

Par ces décisions, le Conseil pose également des jalons qui lui permettront, à l'avenir, d'examiner la constitutionnalité des lois qui lui seront soumises, au regard non seulement du contenu normatif des textes, mais encore de leur procédure d'édiction. À titre d'exemple de censure concrète, on peut citer, même si elle est antérieure aux deux déjà mentionnées, la décision $n^{\circ}$ 2009578 , concernant la loi de mobilisation pour le logement et la lutte contre l'exclusion. Le Conseil censure deux dispositions de la loi ${ }^{23}$, introduites par voie d'amendement, pour n'avoir aucun lien avec le projet de loi déposé ${ }^{24}$. Par là, le Conseil raccroche l'interdiction des cavaliers législatifs avec le principe de clarté et de sincérité du débat parlementaire: la discussion d'un projet de loi ne doit pas, sous peine de nuire au débat nécessaire à l'élaboration de la norme dans une société démocratique, être polluée par l'insertion d'amendements au projet initial n'ayant aucun rapport avec l'objet du texte présenté. Ce rattachement du principe de clarté et de sincérité du débat parlementaire avec l'interdiction des cavaliers législatifs pose finalement la question de l'intérêt de ce nouveau principe, et on imagine mal le Conseil prononcer la censure de dispositions législatives en se fondant uniquement sur le non-respect des temps de paroles des parlementaires en commission.

\section{B. La séparation des pouvoirs}

Le Conseil constitutionnel prend acte de la réforme constitutionnelle du 23 juillet 2008 dans son examen de la loi relative à la communication audiovisuelle et au nouveau service public de la télévision ${ }^{25}$.

16. Par ailleurs, le Conseil détaille, dans le paragraphe 2 commun aux deux décisions, les normes de référence en matière de contrôle des résolutions modifiant les règlements des assemblées : il s'agit de la Constitution, mais également des lois organiques auxquelles elle renvoie, et enfin des mesures législatives prises pour son application.

17. C.C., déc. $\mathrm{n}^{\circ}$ 2006-537 DC du 22 juin 2006, Résolution tendant à modifier le règlement de l'Assemblée nationale, cons. 10.

18. C.C., déc. $n^{\circ} 2009-581$ DC du 25 juin 2009, Résolution tendant à modifier le règlement de l'Assemblée nationale, cons. 28 et 29 . La résolution prévoyait la possibilité pour le président de clore un débat après que quatre orateurs issus de groupes d'opposition ou minoritaires se soient exprimés.

19. Ibid., cons. 20. On observera que le Conseil avait déjà posé une réserve d’interprétation sur la limite du temps de parole dans sa décision $\mathrm{n}^{\circ} 2006-537$ du 22 juin 2006, cons. 10.

20. Ibid., cons. 12

21. Ibid., cons. 34 et 35 , ainsi que 44 , en matière de loi de finances.

22. C.C., déc. $n^{\circ}$ 2009-582 du 25 juin 2009, Résolution tendant à modifier le règlement du Sénat, cons. 30.

23. L'article 115 relatif à l'obligation pour les occupants de tout logement d'y installer un détecteur de fumée; l'article 123 avait pour objet l'extension de certaines parties du CGCT (Code général des collectivités territoriales) aux communes de Polynésie française.

24. C.C., déc. $\mathrm{n}^{\circ}$ 2009-578 du 18 mars 2009, Loi de mobilisation pour le logement et la lutte contre l'exclusion, cons. 30 sq.

25. C.C., déc. Communication audiovisuelle. 
En effet, la nouvelle procédure de nomination des présidents de France Télévisions, Radio France et de la société en charge de l'audiovisuel extérieur de la France, est soumise à l'article 13 de la Constitution ${ }^{26}$, article modifié lors de la révision constitutionnelle de 2008. Désormais, une procédure d'avis de la commission compétente de chaque assemblée est requise en ce qui concerne certaines nominations par le président de la République. Or, la loi déférée prévoit, en sus, un second avis, l'avis conforme du CSA $^{27}$. Les auteurs de la saisine allèguent alors une violation de cet article 13 de la Constitution, qui prévoit la seule consultation des commissions parlementaires compétentes. Néanmoins, le Conseil refuse d'interpréter cette nouveauté comme interdisant «au législateur de fixer ou d'ajouter $[. .$.$] des règles encadrant le pouvoir de$ nomination du président de la République afin de garantir l'indépendance de ces sociétés et de concourir ainsi à la mise en œuvre de la liberté de communication », dans la mesure où elles respectent, notamment, le principe de la séparation des pouvoirs, ce qu'il fait en l'espèce ${ }^{28}$. On peut remarquer à cet égard que ce principe fait l'objet d'une "mobilisation sans précédent», qui montre la volonté du Conseil de réaffirmer son double rôle: celui de « gardien des droits et des libertés dans le cadre de son contrôle de constitutionnalité des lois» mais aussi de garant de «l'équilibre entre les pouvoirs publics » ${ }^{29}$.

La décision $\mathrm{n}^{0}$ 2009-577 Loi relative à la communication audiovisuelle et au nouveau service public de la télévision en est un exemple très révélateur, puisque le Conseil va se fonder à deux reprises sur le principe de la séparation des pouvoirs pour sanctionner des dispositions de ladite loi. Tout d'abord, il l'utilise pour censurer son article 14, qui soumettait cette fois-ci la révocation des présidents à un avis conforme des commissions parlementaires, alors qu'un tel droit n'a été consacré - en vertu de l'article 13 de la Constitution modifié - qu'à l'égard de leurs nominations. Or, cette disposition octroie un droit de veto aux commissions parlementaires, dans la mesure où elles peuvent s'opposer aux nominations du président de la République à la majorité des trois cinquièmes ${ }^{30}$. Elle s'analyse donc comme une dérogation au principe de séparation des pouvoirs, et ne peut être que d'interprétation stricte. Le Conseil a par conséquent censuré la loi sur ce fondement ${ }^{31}$, estimant qu'il s'agissait d'une véritable immixtion du pouvoir législatif dans le pouvoir de l'exécutif au titre des révocations ${ }^{32}$. La possibilité d'une intervention du Parlement dans la procédure de révocation n'est cependant pas rejetée, puisque le Conseil considère qu'un avis simple de ces commissions est suffisamment respectueux du principe de séparation des pouvoirs ${ }^{33}$.

Le Conseil va d'ailleurs donner une portée maximale à ce principe puisqu'il censure une autre disposition - le deuxième alinéa du $3^{\circ}$ de son article 25 de la loi déférée aux motifs qu'elle empiétait sur le domaine réglementaire $^{34}$. Or, il écartait jusque-là cette possibilité, considérant que l'exécutif disposait de moyens d'actions pour assurer cette protection (les procédures des articles 37 alinéa 2 et 41 de la Constitution) ${ }^{35}$; il procédait alors au déclassement des dispositions litigieuses ${ }^{36}$. Cette décision semble donc bien opérer un revirement en la matière, l'empiétement de la loi sur le domaine réglementaire s'analysant dorénavant comme un véritable motif d'inconstitutionnalité ${ }^{37}$.

Cette prise de position est confirmée dans la décision no 2009-579 à propos de la loi organique relative à l'application des articles 34-1,39 et 44 de la Constitution ${ }^{38}$, dans laquelle le Conseil affirme que le " principe de séparation des compétences du pouvoir législatif et du pouvoir réglementaire» empêchait le législateur organique de

26. Le Conseil relève, à cet égard, que le choix de soumettre la nomination à cette procédure visait à "garantir l'indépendance des sociétés nationales de programme et concourir ainsi à la mise en œuvre de la liberté de communication » (C.C., déc. n 2009-576 DC du 3 mars 2009 , Loi organique relative à la nomination des présidents des sociétés France Télévisions et Radio France et de la société en charge de l'audiovisuel extérieur de la France, cons. 4), justifiée par «l'importance de ces emplois pour la garantie des droits et libertés» (C.C., déc. Communication audiovisuelle, cons. 7).

27. Cette compétence relevait auparavant uniquement des pouvoirs du CSA; les auteurs de la saisine invoquaient alors que ce transfert de pouvoir vers l'exécutif porterait atteinte à « la liberté de communication, garantie par l'article 11 de la Déclaration de 1789, l'objectif constitutionnel du pluralisme des courants de pensées et d'opinions ainsi que, par voie de conséquence, les nouvelles dispositions de l'article 34 de la Constitution ». Le Conseil refuse en revanche de le considérer dans la mesure où ce pouvoir du président est soumis à l'avis conforme de ladite autorité (considérant 8 de la décision), garantie suffisante à la préservation de ces droits. Sur ce point, voir R. Ghevontian, S. Lamouroux, O. Le Bot et E. Kerviche, « Jurisprudence du Conseil constitutionnel», p. 590 .

28. C.C., déc. $\mathrm{n}^{\circ}$ 2009-577 DC du 3 mars 2009, cons. 9.

29. L. Baghestani-Perrey, A.-L. Cassard-Valembois et L. Janicot, «Chronique de jurisprudence constitutionnelle $\mathrm{n}^{\circ} 7$ (suite et fin)», p. 10.

30. L'article 13 de la Constitution prévoit en effet que le président ne peut procéder aux nominations si «l'addition des votes négatifs dans chaque commission représente au moins trois cinquièmes des suffrages exprimés au sein des deux commissions ».

31. C.C., déc. $n^{\circ} 2009-577$ DC du 3 mars 2009, cons. 13.

32. R. Ghevontian, S. Lamouroux, O. Le Bot et E. Kerviche, «Jurisprudence du Conseil constitutionnel », p. 595.

33. C.C., déc. ${ }^{\circ}$ 2009-577 DC du 3 mars 2009, cons. 14. Il formule une réserve d'interprétation pour procéder à ce changement de qualification de l'avis, et ainsi rendre la disposition conforme à la Constitution; voir infra en ce qui concerne les réserves d'interprétation.

34. En effet, cette disposition prévoyait la possibilité pour les commissions parlementaires de «formuler un avis sur ce cahier des charges dans un délai de six semaines". Or, " dès lors que le cahier des charges était "fixé" par décret, tout projet de cahier des charges constituait un projet d'acte réglementaire", "Commentaire de la décision $\mathrm{n}^{\circ}$ 2009-577 DC du 3 mars 2009, Loi relative à la communication audiovisuelle et au nouveau service public de la télévision ", Les Cahiers du Conseil constitutionnel, $\mathrm{n}^{\circ}$ 26, août 2009, p. 17-18. Le Conseil en conclut que «la loi déférée fait intervenir une instance législative dans la mise en œuvre du pouvoir réglementaire ; qu'il doit donc être déclaré contraire à la Constitution » (cons. 31).

35. C.C., déc. $n^{\circ} 82-143$ DC du 30 juillet 1982.

36. C.C., déc. $n^{\circ} 2005-512$ DC du 21 avril 2005

37. Sur ce point, voir T. Rambaud et A. Roblot-Troizier, «Chronique de jurisprudence - Droit administratif et droit constitutionnel », RFDA, 2009, p. 580 sq.

38. C.C., déc. $n^{\circ}$ 2009-579 DC du 9 avril 2009, Loi organique relative à l'application des articles 34-1, 39 et 44 de la Constitution. 
soumettre le Gouvernement à une obligation « d'informer le Parlement sur les orientations principales et le délai prévisionnel de publication des dispositions réglementaires qu'il doit prendre dans l'exercice de la compétence exclusive qu'il tient des articles 13 et 21 de la Constitution ${ }^{39}$. Le Conseil confirme donc bien son changement de conception du principe de séparation des pouvoirs, qui s'accompagne dorénavant du principe - de valeur constitutionnelle? - de séparation des compétences.

Cette nouvelle approche du principe de séparation des pouvoirs, qui « rappelle le Conseil constitutionnel des premiers temps, celui qui s'employait avant tout à protéger l'exécutif du Parlement», est perçue comme dangereuse en ce qu'elle serait de nature à «amoindrir la protection des libertés ${ }^{40}$. En effet, dans la décision Communication audiovisuelle, le Conseil refuse de soumettre le pouvoir de révocation du président de la République à l'avis conforme des commissions parlementaires, alors que cette procédure était censée constituer une garantie supplémentaire, visant à protéger la liberté d'expression et, plus particulièrement, l'indépendance des médias (nouvellement consacrée comme objectif de valeur constitutionnelle $)^{41}$. En outre, elle dénature la conception traditionnelle du principe de séparation des pouvoirs et fait fi de «la faculté d'empêcher [qui en] est au cœur » ${ }^{42}$.

Cette évolution s'expliquerait, d'après Lætitia Janicot, par la révision constitutionnelle du 23 juillet 2008, et la volonté du Conseil de donner toute son ampleur aux nouvelles compétences conférées au pouvoir législatif. Cette attitude aboutit néanmoins « à une situation quelque peu paradoxale» puisque le Conseil s'applique à protéger l'exécutif au détriment du Parlement, qui a pourtant vu ses pouvoirs renforcés par le constituant ${ }^{43}$.

Pour ce qui est, au contraire, de l'immixtion du pouvoir réglementaire dans le domaine de la loi, le Conseil rappelle sa jurisprudence traditionnelle qui impose au législateur qui délègue sa compétence au Gouvernement sur le fondement de l'article 38 de la Constitution de limiter cette délégation dans le temps, de prévoir le dépôt d'un projet de loi de ratification et de définir avec précision «la finalité des mesures qu'il se propose de prendre par voie d'ordonnances ainsi que leur domaine d'intervention ${ }^{44}$.

Le Conseil fait encore une application traditionnelle du principe de séparation des pouvoirs, cette fois-ci dans son volet horizontal, dans la décision $n^{\circ}$ 2009-587 Loi organique relative à l'évolution institutionnelle de la NouvelleCalédonie et à la départementalisation de Mayotte ${ }^{45}$. Le Conseil considère en effet que les présidents du Sénat ou de l'Assemblée nationale ne peuvent « décider de réduire le délai de consultation du Congrès de NouvelleCalédonie»sur les propositions de loi comportant des dispositions particulières à la Nouvelle-Calédonie (en vertu de l'article 77 de la Constitution) sans violer ce principe $^{46}$

Enfin, le Conseil veille toujours à ce que la répartition des compétences entre la loi organique, la loi de finances et la loi ordinaire, soit bien respectée. Dans trois de ses décisions relatives à l'examen de lois organiques ${ }^{47}$ et dans deux décisions relatives à l'examen de lois de finances ${ }^{48}$, il relève en effet que certaines de leurs dispositions relèvent du domaine de la loi ordinaire et opère donc leur déclassement. On peut en revanche relever que lorsque c'est au contraire la loi ordinaire qui s'immisce dans le domaine de la loi de finances, le Conseil censure d'office la disposition concernée ${ }^{49}$.

\section{Les réserves d'interprétation du Conseil constitutionnel}

Le Conseil constitutionnel a rendu cette année encore un grand nombre de décisions dans lesquelles il valide la loi ou les dispositions législatives sous couvert de réserves d'interprétation.

Dans sa décision $\mathrm{n}^{0} 2009-590$ du 22 octobre 2009 relative à la Loi sur la protection pénale de la propriété littéraire et artistique sur Internet ${ }^{50}$, le Conseil rappelle que ses prérogatives en matière d'interprétation d'une disposition législative dans le cadre de son contrôle de constitutionnalité de la loi restent relativement limitées ${ }^{51}$. Il ne doit pas se substituer au législateur. Il ne peut interpréter

39. C.C., déc. ${ }^{\circ}$ 2009-579 DC du 9 avril 2009, cons. 16. Il fait également un certain nombre de réserves qui ont toutes pour but de préserver ce principe (voir infra).

40. P. Wachsmann, «La séparation des pouvoirs contre les libertés? », AJDA, 2009, p. 617.

41. Ibid.; L. Baghestani-Perrey, A.-L. Cassard-Valembois et L. Janicot, «Chronique de jurisprudence constitutionnelle $\mathrm{n}^{\circ} 7$ (suite et fin) », p. 11.

42. P. Wachsmann, «La séparation des pouvoirs contre les libertés?».

43. L. Baghestani-Perrey, A.-L. Cassard-Valembois et L. Janicot, «Chronique de jurisprudence constitutionnelle $\mathrm{n}^{\circ} 7$ (suite et fin) », p. 9.

44. C.C., déc. Loi HPST, cons. 22.

45. C.C., déc. $\mathrm{n}^{\circ}$ 2009-587 DC du 30 juillet 2009, Loi organique relative à l'évolution institutionnelle de la Nouvelle-Calédonie et à la départementalisation de Mayotte.

46. C.C., déc. $\mathrm{n}^{\circ}$ 2009-587 DC du 30 juillet 2009, cons. 16.

47. C.C., déc. $n^{\circ}$ 2009-576 DC du 3 mars 2009, cons. 4 ; C.C., déc. $n^{\circ}$ 2009-587 DC du 30 juillet 2009, cons. 8 ; C.C., déc. $n^{\circ} 2009-593$ DC du 19 novembre 2009, Loi pénitentiaire, cons. 8 et 9.

48. C.C., déc. $n^{\circ}$ 2009-599 DC du 29 décembre 2009, Loi de finances pour 2010, cons. 108-111; C.C., déc. nº 2009-600 DC du 29 décembre 2009 , Loi de finances rectificative pour 2009, cons. 12 .

49. C.C., déc. $\mathrm{n}^{\circ}$ 2009-577 DC du 3 mars 2009, cons. 32-34.

50. C.C., déc. $\mathrm{n}^{\circ}$ 2009-590 DC du 22 octobre 2009, Loi relative à la protection pénale de la propriété littéraire et artistique sur Internet (ci-après déc. Hadopi II).

51. Le Conseil a déjà eu l'occasion de préciser l'étendue de son pouvoir d'interprétation dans plusieurs décisions, et notamment dans sa décision nº $2001-$ 455 DC du 12 janvier 2002, Loi de modernisation sociale (cons. 9), et dans sa décision n ${ }^{\circ} 2004-509$ DC du 13 janvier 2005, Loi de programmation pour la cohésion sociale (cons. 25). 
une disposition législative que dans la mesure où cela s'avère nécessaire au contrôle qu'il est appelé à effectuer. En l'espèce, le Conseil estime que c'est au juge judiciaire d'apprécier au cas par cas «si un supplément d'enquête ou d'instruction est nécessaire», et si les éléments de preuve sont suffisants pour décider de la culpabilité des personnes mises en cause, et de déterminer leur peine. Ainsi, le Conseil constitutionnel n'est ni co-législateur, ni juge de l'espèce. Il rappelle que son rôle est strictement limité. Pour autant, on constate dans bon nombre de décisions que la frontière est parfois mince entre le strict contrôle de constitutionnalité de la loi et le travail législatif lorsqu'il utilise la technique de la réserve d'interprétation.

En effet, dans la plupart de ses réserves, le Conseil vient préciser, voire compléter, le travail législatif. C’est ce qu'il fait dans sa décision $\mathrm{n}^{\circ}$ 2009-595 du 3 décembre 2009 sur la loi organique relative à l'application de l'article 61-1 de la Constitution ${ }^{52}$. Le législateur organique dispose, à l'article 23-3 de la loi, que la Cour de cassation peut statuer définitivement sur un pourvoi alors même que le Conseil constitutionnel n'a pas encore statué sur la question prioritaire de constitutionnalité lorsque «l'intéressé est privé de liberté à raison de l'instance et que la loi prévoit que la Cour de cassation statue dans un délai déterminé». Cette disposition n'est constitutionnelle que dans la mesure où le justiciable a la possibilité de réintroduire une instance afin qu'il soit tenu compte de la décision du Conseil constitutionnel. Sous cette réserve, le juge constitutionnel complète substantiellement la loi: le législateur aurait pu lui-même rédiger cette phrase à la fin de l'article contesté. On parle de réserve d'interprétation constructive ${ }^{53}:$ le Conseil outrepasse en quelque sorte son rôle, rôle dont il s'évertue pourtant lui-même à rappeler les limites. La même réserve est faite au considérant 23 de la décision concernant la dernière phrase de l'article 23-5 de la loi relative à l'urgence dans laquelle doivent parfois statuer le Conseil d'État et la Cour de cassation.

Le Conseil constitutionnel revient davantage à son rôle tel qu'il le définit strictement, lorsqu'il se contente, au considérant 28 de la même décision, d'indiquer que les dispositions des articles 23-4 à 23-7 de la loi, faisant référence à la procédure mise en œuvre devant les juridictions suprêmes, doivent s'interpréter comme prescrivant les exigences du droit à un procès équitable, et que, si besoin est, le pouvoir réglementaire apportera les compléments procéduraux nécessaires. La réserve est ainsi simplement directive à l'égard des autorités chargées de l'application de la loi.

Le juge constitutionnel procède de manière identique dans un certain nombre d'autres décisions. Dans celle du 19 novembre 2009 relative à la loi pénitentiaire ${ }^{54}$, le Conseil adresse une réserve directive au pouvoir réglementaire : le décret qui définira les sanctions disciplinaires ne devra pas porter atteinte aux droits et libertés dont les personnes bénéficient dans les limites inhérentes aux contraintes de la détention ${ }^{55}$. Dans celle du 10 juin 2009 relative à la loi favorisant la diffusion et la protection de la création sur Internet ${ }^{56}$, il adresse une réserve directive aux magistrats de l'ordre judiciaire : saisis par des titulaires du droit d'auteur ou de droits voisins, les juges ne devront prononcer «que les mesures strictement nécessaires à la préservation » ou à la cessation d'une atteinte aux droits en cause et cela dans le respect de la liberté d'expression et de communication $^{57}$.

Enfin, dans sa décision Communication audiovisuelle ${ }^{58}$, c'est au législateur qu'il adresse une réserve. Les requérants contestaient la suppression législative des ressources financières de France Télévisions sans prévoir de financement de substitution pour garantir l'indépendance du service public de l'audiovisuel. Ils se plaçaient sur le terrain de l'incompétence négative. Le Conseil relève que le législateur s'est engagé au dernier alinéa du VI de l'article 53 de la loi du 30 septembre 1986, dans sa rédaction résultant de la loi déférée, à ce que chaque loi de finances définisse le montant d'une compensation financière de l'État. L'engagement du législateur se trouve en quelque sorte "constitutionnalisé " ${ }^{59}$ par l'explicitation opérée par le juge constitutionnel sous couvert d'une réserve d'interprétation : « dans le respect de l'indépendance de France Télévisions, il incombera donc à chaque loi de finances de fixer le montant de la compensation financière par l'État de la perte de recettes publicitaires de cette société afin qu'elle soit à même d'exercer les missions de service public qui lui sont confiées. ${ }^{60}$

Le législateur n'a ainsi méconnu ni l'étendue de sa compétence, ni les exigences résultant de l'article 11 de la DDHC. À l'avenir, le Conseil pourrait donc être amené à contrôler une loi allouant à l'audiovisuel public un financement. Mais sera-t-il réellement en mesure d'opérer ce contrôle quant au caractère suffisant ou non du montant alloué ${ }^{61}$ ?

52. C.C., déc. $\mathrm{n}^{\circ}$ 2009-595 DC du 3 décembre 2009, Loi organique relative à l'application de l'article 61-1 de la Constitution (voir infra).

53. Le Conseil utilise cette technique lorsque «la loi n’a pas prévu de dispositions suffisamment précises et que ces omissions portent atteinte à des garanties fondamentales constitutionnellement protégées ». Voir G. Drago, Contentieux constitutionnel français, Paris, PUF, 1998, p. 419-420.

54. C.C., déc. nº 2009-593 DC du 19 novembre 2009, Loi pénitenciaire (ci-après déc. Loi pénitenciaire).

55. C.C., déc. Loi pénitentiaire, cons. 6.

56. C.C., déc. Hadopi I.

57. C.C., déc. Hadopi I, cons. 38.

58. C.C., déc. Communication audiovisuelle.

59. O. Le Bot, «Jurisprudence du Conseil constitutionnel», RFDC, $\mathrm{n}^{\circ}$ 79, 2009/3, p. 595.

60. C.C., déc. $\mathrm{n}^{\circ}$ 2009-577 DC du 3 mars 2009, cons. 19.

61. O. Le Bot, «Jurisprudence du Conseil constitutionnel», p. 596. 
Quant aux réserves émises dans la décision Loi $H P S T^{62}$, elles permettent de valider la philosophie générale du texte, pourtant contestable en termes de libéralisation du système de santé.

En premier lieu, le Conseil déclare conforme le principe d'une participation accrue de personnes privées au service public de la santé à l'alinéa 11 du préambule de la Constitution et au principe de continuité du service public mais en précisant le rôle de l'Agence régionale de santé : lorsque celle-ci définira les modalités de la participation des personnes privées au service public de la santé en la coordonnant avec l'activité des établissements publics, elle devra veiller à ce qu'elles assurent l'exercice continu des missions du service public hospitalier pris dans son ensemble. Le juge semble ainsi vouloir atténuer les conséquences de la libéralisation du système de santé en préservant autant que possible le lien avec la mission de service public originelle. On peut lire cette réserve, mais aussi l'ensemble de la décision, comme une reconnaissance implicite des risques et dérives possibles de la réorganisation du système de santé. L'alinéa $11 \mathrm{du}$ préambule de 1946 y apparaît comme un garde-fou dans un système qui se libéralise de plus en plus.

Par ailleurs, si le principe de la nomination de nonfonctionnaires dans les établissements publics de santé est jugé conforme, le Conseil émet une double réserve directive. Tout d'abord, le pouvoir réglementaire devra fixer des règles permettant l'égal accès des candidats à ces emplois et préciser les modalités permettant d'examiner leurs aptitudes. Ensuite, les autorités compétentes devront fonder leur décision de nomination sur la capacité des intéressés à remplir leur mission.

Le Conseil constitutionnel décide que la Loi HPST ne porte pas atteinte à la protection de la santé garantie par l'alinéa 11 du préambule de 1946: la rénovation du système de santé est conforme au principe constitutionnel. Le système lui apparaît conforme dès lors qu'il ne remet pas en cause le droit aux prestations de soins et l'égal accès de tous aux soins. Pour autant, à la façon du Conseil d'évacuer certaines questions et aux réserves d'interprétation émises, le raisonnement du juge sur la base du préambule de 1946 semble se rapprocher de ses limites. Est-il possible de parler d'un libéralisme économique sous surveillance? Peut-on constater la transformation substantielle du service public de santé validée, mais peu assumée par le Conseil qui se retranche derrière le sacro-saint principe de protection de la santé?

\section{Le contrôle substantiel}

\section{A. Le principe d'égalité}

\section{Le principe d'égalité et la " contribution carbone "}

Le principe constitutionnel d'égalité est régulièrement utilisé par les requérants devant le Conseil constitutionnel pour réclamer la censure de la $1 \mathrm{loi}^{63}$. S'il est souvent invoqué au motif que la loi est exceptionnellement applicable à tous dans les mêmes termes, il conduit rarement le Conseil à la censure ${ }^{64}$. La jurisprudence constitutionnelle de 2009 ne fait pas exception en la matière. Toutefois, dans sa décision sur la loi de finances pour 2010, plus connue sous le nom de "décision taxe carbone " ${ }^{65}$, le Conseil va déclarer certaines dispositions de la loi non conformes au principe d'égalité, en opérant un raisonnement ambitieux, susceptible d'élargir son champ de censure.

Selon une jurisprudence constante du Conseil, il n'est possible de déroger au principe d'égalité qu'au regard de deux conditions alternatives, complétées par une condition cumulative ${ }^{66}$. Le législateur ne peut ainsi rompre l'égalité qu'en cas de situations différentes ou au nom de l'intérêt général, si et seulement si cette différence de traitement est en rapport avec l'objet de la loi ${ }^{67}$. Ces conditions classiques encadrant le contrôle du principe d'égalité sont reprises par le Conseil dans sa décision du 29 décembre 2009. Il considère ainsi que «le principe d'égalité ne fait pas obstacle à ce que soient établies des impositions spécifiques ayant pour objet d'inciter les redevables à adopter des comportements conformes à l'intérêt général, pourvu que les règles qu'il fixe à cet effet soient justifiées au regard desdits objectifs ${ }^{68}$.

Certains auteurs ont pointé du doigt le caractère aléatoire d'un tel raisonnement, au motif que la notion d'intérêt général est floue et donc susceptible de justifier bon nombre de dérogations au principe d'égalité, luimême emprunt d'incertitudes quant à sa détermination ${ }^{69}$. Peuvent ainsi justifier une limitation de la portée du principe constitutionnel d'égalité des considérations telles

62. C.C., déc. Loi HPST

63. Voir notamment G. Merland, «Le principe d'égalité », Revue administrative, $\mathrm{n}^{\circ} 340,1^{\text {er }}$ juillet 2004, p. 370 : «le principe d'égalité est le droit fondamental dont la violation est le plus souvent invoquée par les requérants à l'appui de leur saisine».

64. Ibid.

65. La contribution carbone devait ainsi permettre de diminuer la production de gaz à effet de serre et ainsi lutter contre le réchauffement climatique. Elle ne devait pas s'appliquer aux entreprises soumises au régime communautaire des quotas d'émission de gaz à effet de serre, mis en place par la directive 2003/87/CE du 13 octobre 2003 établissant un système d'échange de quotas d'émission de gaz à effet de serre dans la communauté.

66. C.C., déc. $\mathrm{n}^{\circ}$ 73-51 DC du 27 décembre 1973, Taxation d’office; C.C., déc. $\mathrm{n}^{\circ}$ 2000-441 DC du 28 décembre 2000, Loi de finances rectificative pour 200o; C.C., déc. $n^{\circ}$ 2003-474 DC du 17 juillet 2003, Loi de programme pour l'outre-mer.

67. C.C., déc. $\mathrm{n}^{\circ}$ 2009-578 DC du 18 mars 2009, Loi de mobilisation pour le logement et de lutte contre l'exclusion, cons. 20. Dans cette décision, le Conseil a jugé contraire au principe d'égalité une disposition instaurant une différence de situation non fondée sur un motif d'intérêt général et sans rapport avec l'objectif de la loi qui était d'attribuer des logements aux plus défavorisés.

68. C.C., déc. $\mathrm{n}^{0}$ 2009-599 DC du 29 décembre 2009, Loi de finances pour 2010, cons. 80 (ci-après déc. Loi de finances 2010).

69. D. Rousseau, «Jurisprudence constitutionnelle 2005 ", RDP, $\mathrm{n}^{\circ} 1,1^{\text {er }}$ janvier 2006, p. 265-266; J.-B. Deneuvillers, « Le contrôle interne de constitutionnalité des lois ", Revue administrative, $\mathrm{n}^{\mathrm{o}} 350,1^{\mathrm{er}}$ mars 2006 , p. 138 
que le souci de sécurité juridique des entreprises et des salariés ${ }^{70}$ qui n'ont pourtant pas valeur constitutionnelle.

Concernant la décision Contribution carbone, la protection de l'environnement apparaît incontestablement comme un motif d'intérêt général ${ }^{71}$. Pour le démontrer, le Conseil va se référer à la Charte de l'environnement en prenant le soin de préciser que «l'ensemble des droits et devoirs définis dans la Charte de l'environnement, ont valeur constitutionnelle ${ }^{72}$. La loi Contribution carbone passe donc avec succès cette première étape du contrôle du Conseil: la protection de l'environnement, objectif d'intérêt général, peut justifier l'instauration d'impositions spécifiques.

Le Conseil entend ensuite procéder au contrôle de "l'adéquation des dispositions critiquées à cet objectif ${ }^{73}$, précision faite qu'il ne va pas contrôler la disposition critiquée par les requérants, en l'occurrence l'article $9^{74}$, mais va exclusivement porter son contrôle sur l'article 7 . Le Conseil va alors vérifier que le régime d'exemption instauré par l'article 7 ne méconnaît pas l'objectif de la loi qui est de «mettre en place des instruments permettant de réduire significativement les émissions ${ }^{75}$ de gaz à effet de serre. Ce régime d'exemption visait les entreprises déjà soumises par le droit communautaire à une obligation sous contraintes financières de diminuer leurs émissions de gaz à effet de serre ${ }^{76}$. Mais, le Conseil va estimer que ce système de quotas n'est en réalité pas efficace, de telle sorte que lesdites entreprises, responsables en grande majorité des émissions de $\mathrm{CO}_{2}$ d'origine industrielle, hors carburant, seraient totalement exonérées de l'obligation de réduire leurs émissions. Le Conseil décide donc de censurer le dispositif de la contribution carbone au motif que le régime d'exception est tel qu'il retire tout intérêt au principe et porte nécessairement atteinte à l'objectif de protection de l'environnement fixé par la loi. Le Conseil semble ici « étendre la nature de son contrôle ${ }^{77}$ en déterminant " quel niveau d'exception est supportable de façon à ne pas déséquilibrer l'impact de la loi et dénaturer celle-ci ${ }^{78}$.

Sans doute le Conseil, en effectuant un tel contrôle dans ses décisions antérieures, aurait abouti à une tout autre conclusion. Cela aurait peut-être été le cas pour la Loi réaffirmant le principe du repos dominical et visant à adapter les dérogations à ce principe dans les communes et zones touristiques et thermales ainsi que dans certaines grandes agglomérations pour les salariés volontaires ${ }^{79}$. L'intitulé même de la loi semble emprunt de contradiction. Est ainsi mise en avant comme objectif premier la réaffirmation du principe du repos dominical, et il ne semble que ce ne soit que pour mieux cacher l'ampleur des régimes dérogatoires qui sont apportés au principe. Or, en l'espèce, le Conseil a validé la loi sans effectuer de contrôle approfondi au regard du principe d'égalité ${ }^{80}$.

Pour revenir sur la décision Contribution carbone, relevons que le Conseil ne s'est pas contenté de développer un raisonnement innovant au regard de ses jurisprudences antérieures, il s'est également démarqué fortement de la logique retenue par la Cour de justice des communautés européennes (CJCE) dans l'affaire Arcelor ${ }^{81}$.

La CJCE, dans cette affaire, a considéré, contrairement au Conseil, que le régime de quotas n'est pas inefficace puisqu'il est susceptible de créer pour les entreprises qui y sont soumises des désavantages notamment pécuniaires par rapport aux entreprises exclues de ce système $^{82}$. La CJCE va encore plus loin en estimant que la différence de traitement ainsi créée n'est pas contraire à l'objectif de protection de l'environnement recherché par le législateur communautaire dans la directive. L'exclusion de plusieurs secteurs d'activités, dont la chimie (3400o installations), du champ d'application du régime de quotas est donc justifiée, sans qu'il y ait lieu, pour la Cour, de rechercher l'existence en droit interne d'un système équivalent de réduction des émissions de $\mathrm{CO}_{2}$. La Cour prend tout de même le soin de rappeler que les entreprises soumises à l'application de la directive représentent près de $50 \%$ des émissions de $\mathrm{CO}_{2}$ au niveau communautaire, alors même qu'elles ne comptent que 10000 installations.

La CJCE valide ainsi la directive en insistant sur le caractère progressif et complexe du système de quotas, caractère que l'on retrouve également dans le fonctionnement de la contribution carbone issue de la loi de finances pour 2010 qui prévoyait l'instauration d'une commission de suivi devant évaluer notamment l'évolution du taux de contribution ${ }^{83}$.

De toute évidence, le raisonnement de la CJCE n'a pas été suivi par le Conseil constitutionnel qui a préféré privilégier une interprétation dite de l'effet utile de

70. C.C., déc. $\mathrm{n}^{\circ}$ 2008-568 DC du 7 août 2008, Loi portant rénovation de la démocratie sociale et réforme du temps de travail. Voir notamment L. BaghestaniPerrey, A.-L. Cassard-Valembois et L. Janicot, "Chronique de jurisprudence constitutionnelle $\mathrm{n}^{\circ} 6$ (premier semestre 2008) ( $1^{\text {re }}$ partie) ", Les Petites Affiches, $\mathrm{n}^{\circ}$ 260, 29 décembre 2008, p. 6-19.

71. C.C., déc. $\mathrm{n}^{\circ}$ 2003-488 DC du 29 décembre 2003, Loi de finances rectificative pour 2003.

72. C.C., déc. Loi de finances 2010 , cons. 79.

73. C.C., déc. Loi de finances 2010 , cons. 81 .

74. L'article 9 du projet de loi concernait le dispositif de redistribution de la contribution carbone.

75. C.C., déc. Loi de finances 2010 , cons. 81

76. Directive $2003 / 87 / \mathrm{CE}$ du 13 octobre 2003 établissant un système d'échange de quotas d'émission de gaz à effet de serre dans la communauté.

77. A. Barilari, «La contribution carbone et le Conseil constitutionnel : une censure annoncée ? ", JCP G, n 3,18 janvier 2010 , p. 42.

78. Ibid.

79. Loi n 2009-974 du 10 août 2009.

80. C.C., déc. Repos dominical.

81. CJCE, 16 décembre 2008, Société Arcelor Atlantique et Lorraine e.a., aff. C-127/07.

82. Ibid., cons. 43 .

83. Article 7, II, du projet de Loi de finances pour 2010, adopté le 18 décembre 2009. 
l'objectif de protection de l'environnement ${ }^{84}$. Le Conseil a ainsi retenu dans sa décision Contribution carbone une approche ambitieuse du principe d'égalité, d'autant plus ambitieuse que ledit principe occupe une place importante dans sa jurisprudence, susceptible d'être renforcée par son contrôle par voie d'exception.

\section{Le principe d'égalité et la question prioritaire de constitutionnalité}

En vue de la mise en place d'un contrôle a posteriori de la constitutionnalité des lois, via la procédure de la question prioritaire de constitutionnalité (QPC), introduite par la Loi organique du 10 décembre 2009 relative à l'application de l'article 61-1 de la Constitution ${ }^{85}$, certains acteurs et auteurs de la vie juridique se sont interrogés sur les principes qui pourraient être le plus souvent invoqués par le justiciable ${ }^{86}$. Les saisines déjà nombreuses invoquant le principe d'égalité pourraient à cet égard se multiplier ${ }^{87}$.

Si l'ouverture de la procédure du contrôle de constitutionnalité à un nombre indéfini de requérants augmentera mécaniquement le nombre de saisines, d'autres explications plus juridiques à ce succès éventuellement surprenant du traditionnel principe d'égalité peuvent être évoquées.

Premièrement, le principe d'égalité figure parmi les droits et libertés constitutionnellement garantis, et son respect est déjà assuré par le contrôle de conventionnalité opéré par les juridictions administratives et judiciaires. Ce faisant, par cette voie, le champ d'application du principe de non-discrimination - homologue du principe d'égalité - est limité. Posé par l'article 14 de la Convention européenne de sauvegarde des Droits de l'homme et des libertés fondamentales, il ne s'applique qu'à la jouissance des droits et libertés reconnus par ladite Convention et ses protocoles additionnels. Aussi, le Conseil d'État, par l'arrêt $M^{m e}$ Bahomar ${ }^{88}$, n'a-t-il pu sanctionner une atteinte au principe d'égalité au titre de la Convention qui suppose l'articulation avec un droit qu'elle protège et que n'a pu invoquer la requérante. Le champ d'application du principe d'égalité en droit constitutionnel français est beaucoup plus large, et si le Conseil avait pu, à l'époque, statuer sur la conformité de la disposition législative en cause dans l'affaire mentionnée, l'issue du litige concernant $\mathrm{M}^{\mathrm{me}}$ Bahomar aurait été vraisemblablement différente ${ }^{89}$.

Secondement, l'article 23-2 de la Loi organique relative à l'application de l'article 61-1 de la Constitution fixant les conditions de recevabilité de la requête dispose, entre autres, que la disposition législative ne pourra être contestée que si «elle n'a pas déjà été déclarée conforme à la Constitution dans les motifs et le dispositif d'une décision du Conseil constitutionnel, sauf changement des circonstances ». Le Conseil précise dans sa décision $n^{\circ}$ 2009595 du 3 décembre 2009 relative à ladite loi organique que cela conduit à ce que, bien que déclarée conforme à la Constitution, une disposition peut être à nouveau examinée si « un réexamen est justifié par les changements intervenus, depuis la précédente décision, dans les normes de constitutionnalité applicables ou dans les circonstances, de droit ou de fait, qui affectent la portée de la disposition législative critiquée ${ }^{90}$. Or, comme l'ont relevé plusieurs auteurs, la référence aux circonstances peut paraître "délicate ${ }^{91}$ puisqu'elle risque de "rendre la déclaration de conformité dans le cadre du contrôle $a$ priori relative et contextuelle ${ }^{92}$, mais également toutes les décisions du Conseil a posteriori - l'écou-lement du temps provoquera à lui seul un changement de circonstances ${ }^{93}$. En outre, la mise en garde nous semble encore plus fondée s'agissant du contrôle de la conformité au principe d'égalité. En effet, le contrôle du respect de ce principe s'opère souvent en s'assurant de la justification d'une différence de traitement à des situations semblables ou différentes, compte tenu des circonstances. L'application du principe d'égalité - à partir du moment où elle ne repose pas sur une conception exclusivement formelle de l'égalité ${ }^{4}$ - nous semble intrinsèquement circonstanciée, si bien que toute nouvelle affaire pourrait justifier un nouvel examen par le Conseil constitutionnel.

Pour s'en convaincre, il convient de faire état de l'application du principe d'égalité par le Conseil dans les décisions qui font l'objet de cette chronique. L'édifice jurisprudentiel relatif à ce principe révèle que le Conseil n'a pas de conception univoque de l'égalité. Celle-ci peut, d'une part, être entendue de manière formelle et impliquer un traitement uniforme des sujets de droit ou, d'autre part, recouvrir une signification matérielle et permettre de tenir compte de la similitude ou de la disparité des

84. P. Billet, «L'invalidation de la contribution carbone », JCP A, $\mathrm{n}^{\circ}$ 4, 25 janvier 2010, p. 35 .

85. Loi $n^{\circ}$ 2009-1523 du 10 décembre 2009 relative à l'application de l'article 61-1 de la Constitution.

86. Cette question était la première adressée aux personnalités auditionnées à l'Assemblée nationale par la Commission des lois constitutionnelles, de la législation et de l'administration générale de la République; auditions ouvertes à la presse sur le projet de loi organique relatif à l'application de l'article 61-1 de la Constitution.

87. La plupart des membres auditionnés ont évoqué, en premier lieu, le principe d'égalité.

88. CE, 7 février $2008, \mathrm{n}^{\circ} 267744$.

89. J.-M. Sauvé, audition à l'Assemblée nationale, 23 juin 2009; et le commissaire du Gouvernement J.-P. Thellay d'ajouter qu’il avait pu «paraître curieux qu'un requérant arrive à convaincre du caractère discriminatoire d'une disposition, sans que l'invocation de la Convention européenne des Droits de l'homme lui permette de parvenir à ses fins ", conclusions sous Conseil d'État, section, 7 février 2008, M ${ }^{m e}$ veuve Rahba Bahomar, RFDA, 2008 , p. 516

90. C.C., déc. Loi organique 61-1, cons. 13 .

91. B. Mathieu, audition à l'Assemblée nationale, 23 juin 2009.

92. P. Bon, «La question prioritaire de constitutionnalité après la loi organique du 10 décembre 2009 », RFDA, 2009 , p. 1117.

93. Ibid.

94. A. Frank, «Les critères objectifs et rationnels dans le contrôle constitutionnel de l'égalité », RDP, $\mathrm{n}^{\circ}$ 1, 2009, p. 77. 
situations - autrement dit, et à notre sens, des circonstances - pour justifier un traitement différencié par le législateur.

Sans recourir à la prise en compte des situations, le Conseil, dans la décision $\mathrm{n}^{\circ}$ 2009-594 relative à la Loi HPST, s'est prononcé sur le principe d'égalité à deux reprises ${ }^{95}$. En premier lieu, il considère qu'une disposition législative, permettant aux établissements de santé de conclure des contrats avec l'Agence régionale de santé, ne conduit pas à une rupture d'égalité entre usagers du service public, dans la mesure où ces établissements « seront tenus de garantir l'accès de tous aux soins de qualité et d'en assurer la prise en charge aux tarifs et honoraires réglementés ${ }^{96}$. En second lieu, le recrutement de directeurs d'hôpitaux, par d'autres voies que celles du concours, ne présente pas de rupture d'égalité à l'endroit du personnel, tant qu'une autre méthode de recrutement garantit également une parfaite égalité des chances entre les candidats ${ }^{97}$. Dans les deux cas ci-dessus, selon le Conseil, les modalités d'organisation du service public de santé ne risquent pas d'entraîner de résultats inégalitaires tant à l'égard des usagers qu'à celui de son personnel. Le Conseil exerce un contrôle in abstracto du respect du principe d'égalité et n'a donc pas recours à l'appréciation des circonstances.

En outre, dans la décision n ${ }^{\circ}$ 2009-594 du 3 décembre 2009 relative à la Loi portant régulation des transports ferroviaires, la non-dissociation des activités commerciales et des activités de gestion de réseau n'entraîne pas de rupture d'égalité entre les acteurs économiques. En effet, parce que « la loi n'a pas pour objet d'organiser, avant ces échéances, l'ouverture de la concurrence de ces services de transports ${ }^{98}$, le moyen invoqué par les requérants est inopérant. Autrement dit, aujourd'hui et jusqu'à l'échéance des contrats en cours, fixée à quinze ans, le législateur n'entend pas assurer l'égalité des acteurs du secteur. Il est donc vain d'invoquer, pour l'instant, une rupture du principe d'égalité. Celle-ci pourra néanmoins être appréciée à compter de 2025. Ce faisant, le Conseil, par sa référence aux échéances, entrevoit un changement de circonstances et relativise la portée de sa décision.

La prise en compte des circonstances et, plus précisément, de la différence de situations auxquelles vont s'appliquer les dispositions législatives, reflète une conception plus matérielle de l'application du principe d'égalité. Aussi le Conseil constitutionnel, par la décision $\mathrm{n}^{\circ}$ 2009-588 du 6 août 2009 relative au repos dominical, reconnaît-il que la loi entraîne une rupture d'égalité, mais il la justifie au regard de la différence de situations entre salariés travaillant en "zone touristique» et ceux travaillant en zone de " consommation exceptionnelle» ${ }^{99}$. En revanche, parce qu'entre Paris et les autres agglomérations, " aucune différence de situation ne justifie que le pouvoir » de délimiter les zones susceptibles d'être considérées comme «zones touristiques » soit confié, dans un cas, au préfet et, dans l'autre, à un élu, le Conseil sanctionne la disposition législative en cause au nom du principe d'égalité ${ }^{100}$

Enfin, il convient d'accorder plus de développement à la décision n ${ }^{0}$ 2009-599 du Conseil relative à la taxe carbone ${ }^{101}$ dans la mesure où le juge est très minutieux dans l'appréciation des circonstances de l'espèce. De manière générale, le principe d'égalité ne s'oppose pas à ce que le législateur règle de façon différente des situations différentes, voire qu'il prévoit, en matière d'impôt, par exemple, l'exonération totale de certains contribuables, tant que la différence de traitement constatée est fondée sur des critères objectifs et rationnels au regard de l'objet de la loi ${ }^{102}$. Or, après avoir considéré la somme des exemptions totales instituées par l'article 7 de la loi de finances pour 2010 prévoyant l'introduction d'une nouvelle « taxe carbone » dans le paysage fiscal français, le Conseil constate que ces exonérations sont contraires à l'objectif de la loi et que, dès lors, la rupture caractérisée de l'égalité devant les charges publiques, nécessairement entraînée par des dérogations au paiement de la taxe, ne peut être justifiée. Cette décision est tout à fait conforme à l'édifice jurisprudentiel en la matière ${ }^{103}$. Cependant, elle semble marquer un nouveau pas dans l'application du principe d'égalité dans la mesure où, pour sanctionner le dispositif, à l'aune des exonérations prévues par la loi, le Conseil évoque des chiffres et notamment la part d'émissions de dioxyde de carbone d'origine industrielle - $93 \%$ - dont sont responsables les acteurs industriels épargnés par la nouvelle taxe. On peut se demander après que le Conseil a statué de cette manière approfondie, si la réduction ne serait-ce que de quelques points - de cette part évoquée ci-dessus ne provoquerait pas un changement de circonstances de fait justifiant un nouvel examen via la question prioritaire de constitutionnalité.

\section{B. La protection du droit d'auteur et l'accès à Internet}

Depuis la Loi $n^{o}$ 2004-204 du 9 mars 2004 portant adaptation de la justice aux évolutions de la criminalité, le délit de contrefaçon réprimé à l'article L. 335-2 du Code de la propriété intellectuelle (CPI) est puni de trois ans

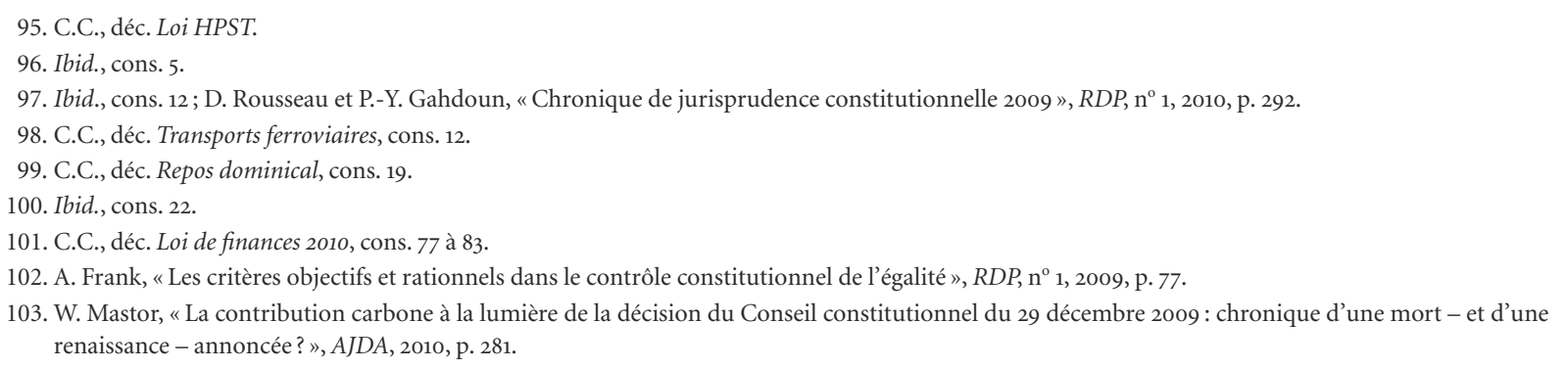


d'emprisonnement et 300000 euros d'amende. Dès 1992, "toute reproduction, représentation ou diffusion, par quelque moyen que ce soit, d'une œuvre de l'esprit en violation des droits de l'auteur» (art. L. 335-3 CPI) est considérée comme une contrefaçon. À ce titre sont donc punissables les téléchargements et échanges par Internet de fichiers vidéo et musicaux en violation des droits d'auteur. Toutefois, les juges hésitent très largement à sanctionner à cette hauteur la contrefaçon dite « domestique », voire ne sanctionnent pas du tout ${ }^{104}$.

Par le projet de Loi favorisant la diffusion et la protection de la création sur Internet, adopté définitivement en deuxième lecture par le Sénat le 13 mai 2009, le Gouvernement et le législateur souhaitaient notamment et surtout régler ce " problème » de la contrefaçon domestique par Internet. Dans un discours en date du 23 novembre 2007, Nicolas Sarkozy affirmait déjà qu' «Internet ne doit pas être un "Far West" high-tech, une zone de non-droit où des "hors-la-loi" peuvent piller sans réserve les créations ${ }^{105}$. La solution choisie fut de dépénaliser la question et de renvoyer la possible sanction à une Autorité administrative indépendante (AAI) ${ }^{106}$, la Haute Autorité pour la diffusion des œuvres et la protection des droits sur Internet (HADOPI), instituée en remplacement de l'ancienne Autorité de régulation des mesures techniques créée par la Loi DADVSI en $2006^{107}$.

Cette Haute Autorité est composée de deux organes, un collège et une commission de protection des droits. Le projet de loi confiait à cette dernière le pouvoir de constater et de sanctionner tout manquement à la nouvelle obligation de surveillance de sa connexion à Internet créée par son article $11^{108}$. Il prévoyait ainsi une riposte en deux temps en cas de manquement constaté : 1) Une recommandation envoyée à l'abonné lui intimant de surveiller son accès ; 2) Une possibilité de sanction en cas d'un deuxième manquement enregistré dans l'année. Cette sanction intervenait après une procédure contradictoire et, en fonction de la gravité des manquements, pouvait consister en : une suspension de deux mois à un an de l'accès à Internet avec interdiction de souscrire un contrat du même type auprès d'un autre opérateur et une injonction de prendre des mesures de nature à prévenir le renouvellement du manquement constaté, notamment par l'installation d'un moyen de sécurisation figurant sur une liste définie par la loi. L’abonné ne pouvait éviter la sanction que dans trois cas: s'il avait déjà installé un moyen de sécurisation, s'il démontrait l'usage frauduleux de sa connexion ou en cas de force majeure.

Cette obligation de surveillance, face à un téléchargement illégal pourtant toujours soumis au régime de la contrefaçon, est considérée par le Conseil comme distincte dudit délit de contrefaçon ${ }^{109}$. Ce n'est donc pas le téléchargement illégal de contenu protégé en tant que tel qui est ici sanctionné mais le fait, pour un abonné à Internet, de n'avoir pas tout mis en œuvre pour empêcher ce type de téléchargement à partir de sa connexion. Cela ressemble fort à tenter de passer par la fenêtre quand les juges refusent d'emprunter la porte.

Saisi de cette loi par plus de soixante députés, le Conseil constitutionnel a censuré ce dispositif de sanction administrative comme contraire à la Constitution, et notamment aux articles 9 et 11 de la DDHC ${ }^{110}$.

Reprenant un considérant de principe posé dès $1999^{111}$, le Conseil constitutionnel a ainsi considéré que le renversement de la charge de la preuve en la matière instaurait « une présomption de culpabilité à l'encontre du titulaire de l'accès à Internet, pouvait conduire à prononcer contre lui des sanctions privatives ou restrictives de droit ${ }^{112}$. Dans sa décision $n^{0} 99-411$ DC du 16 juin 1999, le Conseil avait reconnu valeur constitutionnelle au principe selon lequel, en matière de peines et de sanctions, nul n'est punissable que de son propre fait ${ }^{113}$. Ainsi, une présomption de culpabilité doit-elle être simple et aisément renversable. En l'espèce, vu la difficulté pour un abonné de démontrer que sa connexion Internet a été piratée, le Conseil a choisi de censurer cette disposition.

Toutefois, le Conseil n'avait pas besoin de préciser ce motif de censure puisqu'il venait déjà de constater

104. Voir J. Cedras, Le téléchargement illicite d'œuvres protégées par le droit d'auteur. Rapport à monsieur le ministre de la Culture et de la Communication, avril 2007. Par exemple, TGI de Paris, 21 juin 2006, Pathé Renn Production et autres c. 9 Telecom Réseau et autres; TGI de Rennes, 30 novembre 2006, Scpp, Sppf c. Anne-Sophie L.; TGI de Nantes, 11 janvier 2007, Syndicat de l'édition vidéo et autres c. Cynthia C., Sébastien G. ; CA de Versailles, 16 mars 2007, Alain O. c. Sacem et autres.

105. Nicolas Sarkozy, Discours prononcé à l’occasion du Rapport Olivennes sur le téléchargement illégal, disponible sur : www.numerama.com/magazine/5691-urgent-le-discours-de-nicolas-sarkozy-accord-olivennes.html, vu le 10 mars 2010.

106. La loi, en son article 5, parle d'une Autorité publique indépendante que le Conseil constitutionnel a requalifiée dans sa décision d'Autorité administrative indépendante (C.C., déc. Hadopi I, cons. 4).

107. Loi relative au droit d'auteur et aux droits voisins dans la société de l'information, 3 août 2006.

108. Cet article imposait à toute "personne titulaire de l'accès à des services de communication au public en ligne [...] l'obligation de veiller à ce que cet accès ne fasse pas l'objet d'une utilisation à des fins de reproduction, de représentation, de mise à disposition ou de communication au public d'œuvres ou d'objets protégés par un droit d'auteur ou par un droit voisin sans l'autorisation des titulaires des droits prévus».

109. C.C., déc. Hadopi I, cons. 7.

110. Des articles protégeant respectivement la présomption d'innocence et la liberté de communication.

111. C.C., déc. 99-411 DC du 16 juin 1999, cons. 5: «aux termes de l'article 9 de la Déclaration des Droits de l’homme et du citoyen : “Tout homme étant présumé innocent jusqu'à ce qu'il ait été déclaré coupable, s'il est jugé indispensable de l’arrêter, toute rigueur qui ne serait pas nécessaire pour s'assurer de sa personne doit être sévèrement réprimée par la loi”; qu’il en résulte qu'en principe le législateur ne saurait instituer de présomption de culpabilité en matière répressive ; que, toutefois, à titre exceptionnel, de telles présomptions peuvent être établies, notamment en matière contraventionnelle, dès lors qu'elles ne revêtent pas de caractère irréfragable, qu'est assuré le respect des droits de la défense et que les faits induisent raisonnablement la vraisemblance de l'imputabilité. »

112. C.C., déc. Hadopi I, cons. 18.

113. Voir également C.C., déc. $n^{\circ}$ 2004-496 DC du 10 juin 2004 à propos de la responsabilité des hébergeurs de sites Internet. 
l'inconstitutionnalité de l'ensemble de cette procédure «administrative» de sanction. En effet, au terme d'une conciliation entre la liberté de communication, d'une part, et le droit de propriété, d'autre part, le Conseil a considéré que le législateur ne pouvait confier de tels pouvoirs à une autorité administrative indépendante ${ }^{114}$. Pour arriver à cette conclusion, il a, dans un premier temps, explicitement reconnu une liberté d'accéder aux moyens de communication au public en ligne (ou Internet), déduite de la liberté de communication. Il a ensuite rappelé le caractère fondamental pour la démocratie de l'exercice de cette liberté ${ }^{115}$. Ainsi, le Conseil peut affirmer que les atteintes portées à son exercice doivent être nécessaires, adaptées et proportionnées à l'objectif poursuivi ${ }^{116}$. Il inscrit donc son action dans un plein contrôle de proportionnalité ${ }^{117}$.

Ayant reconnu depuis 2004 la «sauvegarde de la propriété intellectuelle ${ }^{118}$ et son pendant, la «lutte contre les pratiques de contrefaçon sur Internet ${ }^{119}$, comme un objectif d'intérêt général ${ }^{120}$, le Conseil a validé la nécessité de mesures de protection. En revanche, il semble que ce soit le caractère « adapté » de la procédure qui lui pose problème. Le fait que toute personne puisse se voir restreindre l'exercice «de son droit de s'exprimer et de communiquer librement, notamment depuis son domicile ", sans aucune intervention préalable de l'autorité judiciaire, ne serait ainsi pas adapté « à la nature de la liberté garantie par l'article 11 de la Déclaration de $1789{ }^{121}$. Selon Aurélie Binet-Grosclaude, cette décision revient donc implicitement à constater l'existence de libertés supérieures aux autres et qui nécessitent dans ce cas l'intervention du juge judiciaire. Ce dernier devient ici le gardien de la liberté d'expression ${ }^{122}$.

Après le passage du Conseil, il ne reste plus rien du pouvoir de sanction pour défaut de surveillance de sa connexion à Internet conféré par le projet de loi à la Commission des droits de la HADOPI. Le législateur a donc tenu compte de cette décision lors de l'examen du projet de Loi relative à la protection pénale de la propriété littéraire et artistique sur Internet.
Dès le titre du projet de loi, tout un chacun peut comprendre que l'on a changé de registre. D'une sanction confiée à une autorité administrative, nous sommes revenus à une punition par le droit pénal.

La première nouveauté de cette loi est l'instauration d'une nouvelle peine complémentaire de suspension de l'accès à un service de communication au public en ligne. Cette peine pourra être associée tant au délit de contrefaçon prévu aux articles L. 335-2, L. 335-3 et L. 335-4 CPI, qu'aux contraventions de la cinquième classe prévues par le CPI. Dans ce dernier cas, la peine complémentaire ne pourra être appliquée qu'en cas de «négligence caractérisée» de l'abonné auquel la commission des droits aura préalablement adressé une « recommandation l'invitant à mettre en œuvre un moyen de sécurisation de son accès à Internet ${ }^{123}$. Il est d'autant plus important de noter que dans les deux cas, l'application d'une telle peine complémentaire «n'affecte pas, par elle-même, le versement du prix de l'abonnement au service » ${ }^{124}$ et que tout utilisateur "suspendu» qui chercherait à contracter un nouvel abonnement auprès d'un autre fournisseur d'accès pourrait se voir condamné à deux ans de prison et 30 ooo euros d'amende au titre de l'article 434-41 du Code pénal modifié par cette loi.

Pour la peine complémentaire associée au délit de contrefaçon, le Conseil rappelle qu'en matière de peine et de sanction, il n'exerce qu'un contrôle de l'erreur manifeste du législateur consistant en une possible « disproportion manifeste entre l'infraction et la peine encourue ${ }^{125}$. Sans aucune justification supplémentaire, le Conseil reconnaît ensuite que l'instauration d'une telle peine complémentaire ne méconnaît donc pas le principe de nécessité des peines.

Dans le cas de celle associée aux contraventions de cinquième classe, rappelant que sa compétence ne s'étend pas à la constitutionnalité des règlements, le Conseil renvoie ainsi aux juridictions compétentes l'examen de la proportionnalité d'une peine qui ne peut s'apprécier qu' « au regard de l'ensemble des éléments constitutifs de l'infraction [définis par le pouvoir réglementaire] qu'elle

114. Ce n'est pas, en soi, le pouvoir de sanction confié à une AAI qui est ici censuré, le Conseil rappelant cette possibilité pour le législateur (C.C., déc. Hadopi I, cons. 14). Voir, en ce sens, C.C., déc. $n^{\circ} 88-248$ DC du 17 janvier 1989, cons. 27 ; C.C., déc. nº 89-260 DC du 28 juillet 1989 , cons. 6 ; C.C., déc. $\mathrm{n}^{\circ}$ 2000-433 DC du 27 juillet 2000, cons. 50 ; C.C., déc. n 2006-540 DC du 27 juillet 2006, cons. 71 . Pour une analyse plus détaillée du pouvoir de sanction pénale des AAI, voir A. Binet-Grosclaude, «La décision du Conseil constitutionnel du 10 juin 2009 relative à la loi favorisant la diffusion et la protection de la création sur Internet: un coup d'arrêt au pouvoir de sanction des AAI ? ", Droit pénal, nº 11, novembre 2009 , p. 17.

115. Voir, en ce sens, au niveau européen, l'arrêt Cour EDH, 7 décembre 1976, aff. Handyside c. Royaume-Uni, $\$ 49$ : «La liberté d'expression constitue l'un des fondements essentiels de pareille société [démocratique], l'une des conditions primordiales de son progrès et de l'épanouissement de chacun. »

116. C.C., déc. Hadopi I, cons. 15. Voir en ce sens également: C.C., déc. nº 2008-562 DC du 21 février 2008 à propos de la liberté individuelle, de la liberté d'aller et venir et du respect de la vie privée. Le Conseil avait déjà dégagé un critère proche de celui du caractère adapté, à savoir l'adéquation d'une mesure : voir en ce sens C.C., déc. ${ }^{\circ}{ }^{2005-528}$ DC du 15 décembre 2005, cons. 15.

117. Voir V. Goesel-Lebihan, «Le contrôle de proportionnalité exercé par le Conseil constitutionnel : présentation générale », Petites Affiches, $\mathrm{n}^{\circ} 46,5$ mars 2009, p. 62-69.

118. C.C., déc. Hadopi I, cons. 13 .

119. C.C., déc. Hadopi I, cons. 15 .

120. C.C., déc. $n^{\circ}$ 2004-499 DC du 29 juillet 2004, cons. 13. La sauvegarde de la propriété intellectuelle est ici directement rattachée au droit de propriété en tant qu'il a vu son champ d'application s'étendre «à des domaines nouveaux» (rappelé par C.C., déc. n 2006-540 DC du 27 juillet 2006, cons. 15).

121. C.C., déc. Hadopi I, cons. 16.

122. A. Binet-Grosclaude, «La décision du Conseil constitutionnel du 10 juin 2009... », p. 18.

123. Article 8 insérant un article L. 335-7-1 dans le CPI.

124. Art. L. 335-7 et L. 335-7-1 CPI

125. C.C., déc. Hadopi II, cons. 20. 
est destinée à réprimer ${ }^{126}$. Tout le problème sera au final de pouvoir juger de la personnalisation de l'infraction principale. Dans un jugement en date du 23 février 2009, le tribunal de Guingamp a ainsi établi que l'adresse IP ne suffit pas pour établir la culpabilité d'un internaute. De même, dans une étude publiée le 9 juin $2008^{127}$, deux professeurs et un étudiant de l'Université de Washington ont pu faire croire que c'était une imprimante qui téléchargeait des fichiers protégés.

Enfin, le Conseil affirme très rapidement au considérant 22 que l'obligation de payer son abonnement par l'utilisateur suspendu «trouve son fondement dans le fait que l'inexécution du contrat est imputable à l'abonné» et ne constitue «ni une peine ni une sanction ayant le caractère d'une punition ». En ce sens, elle ne méconnaît aucune exigence constitutionnelle. Le Conseil avait déjà jugé en mars de la même année que «le prélèvement sur les ressources financières des organismes d'habitations à loyer modéré $[. .$.$] ne constitue [...] pas une sanction$ ayant le caractère d'une punition » car il «n'a pas pour objet de sanctionner le manquement à une obligation fixée par la loi ou le règlement ${ }^{128}$.

Le Conseil semble ici cohérent en la matière même si nous aurions apprécié une justification plus poussée sur la nature d'une peine ou d'une sanction ayant le caractère d'une punition.

Une deuxième nouveauté est la soumission des délits de contrefaçon à la liste des délits jugés par le tribunal correctionnel statuant à juge unique, ainsi que la possibilité pour la victime de demander au juge de statuer par ordonnance pénale. Sur ce point, il est important de noter que l'argumentaire des députés requérants tournait plus autour de la constitutionnalité de la procédure à juge unique que de son application au délit de contrefaçon. Le Conseil balaye facilement les objections en renvoyant à sa décision du 29 août 2002 où il avait pu en juger ${ }^{129}$. Il ne censure au final que l'incompétence négative du législateur en ce sens qu'il n'a pas bien prévu le régime de l'opposition possible de la victime à l'ordonnance pénale dans le cadre de la procédure simplifiée ${ }^{130}$.

126. Ibid., cons. 28.

127. M. Piatek, T. Kohno et A. Krishnamurthy, «Challenges and directions for monitoring $\mathrm{P}_{2} \mathrm{P}$ file sharing networks - or - Why my printer received a DMCA takedown notice», University of Washington Technical Report, UW-CSE-08-06-01, 9 juin 2008 :

http://dmca.cs.washington.edu/uwcse_dmca_tr.pdf.

128. C.C., déc. $n^{\circ}$ 2009-578 DC du 18 mars 2009, cons. 4.

129. C.C., déc. $n^{\circ} 2002-461$ DC du 29 août 2002.

130. C.C., déc. Hadopi II, cons. 14. 
